\title{
EFEITOS DA CARGA ANTICOLINÉRGICA COGNITIVA EM IDOSOS - UMA REVISÃO INTEGRATIVA
}

\section{Caroline Ribeiro Borja-Oliveira}

resumo

Idosos são mais susceptíveis aos efeitos adversos cognitivos de fármacos com atividade anticolinérgica. Isso se deve a fatores associados à velhice, como comprometimento da atividade colinérgica central, aumento da permeabilidade da barreira hematoencefálica, redução do metabolismo hepático e da excreção renal e polifarmácia. A escala de Carga Anticolinérgica Cognitiva (ACB) foi elaborada com o intuito de reduzir a ocorrência eventos adversos cognitivos à farmacoterapia, como declínio cognitivo, demência e delírio. O objetivo desta revisão integrativa foi reunir achados obtidos em estudos clínicos sobre a associação entre alterações cognitivas e ACB por meio da escala. A busca nas bases MEDLINE/PubMed e BVS-Bireme gerou 11 artigos completos em inglês. Sete são longitudinais, três transversais e um observacional. Oito foram conduzidos nos Estados Unidos. Nove de 10 evidenciaram a relação entre ACB e déficit cognitivo. O MMSE foi o teste de avaliação cognitiva mais usado. As evidências mostram a importância da revisão da farmacoterapia de todo paciente com déficit cognitivo ou delírio. Deve-se evitar

1 Graduada em Farmácia. Pós-doutora em Farmacologia. Docente dos cursos de graduação e de pós-graduação em Gerontologia da Universidade de São Paulo. E-mail: cborja@usp.br. 
assumir que o declínio cognitivo é demência até as possíveis causas farmacológicas tenham sido descartadas.

palavras-chave

Antagonistas Colinérgicos. Efeitos Colaterais e Reações Adversas Relacionados a Medicamentos. Delirium. Demência. Transtornos Cognitivos. Saúde do Idoso.

\section{Introdução}

O uso concomitante de múltiplos medicamentos e as alterações fisiológicas do envelhecimento tornam os idosos mais propensos à toxicidade farmacológica e ao desenvolvimento de efeitos adversos cognitivos (FARRELL; EISENER-PARSCHE; DALTON, 2014). No sistema nervoso central, fármacos anticolinérgicos bloqueiam receptores muscarínicos e nicotínicos, que estão envolvidos no controle de funções cognitivas como memória, aprendizado e atenção (ALBUQUERQUE et al., 2009; VENTURA, et al., 2010).

Mesmo que se tenha conhecimento de que alguns fármacos anticolinérgicos não alcançam o sistema nervoso central (SNC), o envelhecimento está associado a alterações da barreira hematoencefálica que resultam em aumento da permeabilidade e penetração facilitada de moléculas no cérebro (SHAH et al., 2013). O aumento da permeabilidade da barreira hematoencefálica associado ao envelhecimento em indivíduos saudáveis está bem documentado (FARRALL; WARDLAW, 2009; POPESCU et al., 2009).

Visando contribuir com a identificação de fármacos com propriedades anticolinérgicas e, desse modo, com a redução do risco de eventos adversos cognitivos, como declínio cognitivo, demência e delírio, Boustani e colaboradores (2008) desenvolveram a "Anticholinergic Cognitive Burden scale" ou escala de Carga Anticolinérgica Cognitiva (ACB) (vide Anexo). A elaboração da escala ACB se deu em etapas. Na primeira, os autores obtiveram, por meio de uma revisão de 80 estudos científicos publicados entre 1966 e 2007, evidências sobre a relação entre exposição a fármacos anticolinérgicos e déficit cognitivo. A revisão gerou uma lista que incluía certos medicamentos vendidos sob prescrição médica e também alguns isentos de prescrição. Essa lista foi compartilhada com uma equipe interdisciplinar de experts, composta por geriatras, farmacêuticos, psiquiatras, clínicos, enfermeiros e neurocientistas. 
Com base nas evidências encontradas na revisão, o grupo selecionado por Boustani et al. (2008) categorizou a atividade anticolinérgica cognitiva dos medicamentos em "possível" e "definida". Esta última foi subdividida em "moderada" e "severa". Tal classificação permitiu ao grupo estabelecer um sistema de escore.

Conforme esse sistema, os fármacos destituídos de evidências clínicas quanto a efeitos adversos cognitivos que, porém, apresentaram atividade anticolinérgica sérica ou afinidade in vitro por receptores muscarínicos, foram enquadrados na categoria "possível" e receberem escore 1. Já os fármacos que possuíam evidências clínicas relevantes e estabelecidas quanto a efeitos adversos anticolinérgicos cognitivos foram enquadrados nas categorias "moderada" e "severa", recebendo, respectivamente, escores 2 e 3. Essa divisão, entre as categorias "moderada" e "severa", foi estabelecida conforme a habilidade de atravessar a barreira hematoencefálica e a associação com o desenvolvimento de delírio. Para os medicamentos destituídos de qualquer ação anticolinérgica relatada, atribui-se escore zero, de modo que a pontuação total resultante da soma dos escores dos fármacos utilizados por um mesmo indivíduo revele a carga anticolinérgica cognitiva à qual o mesmo está sujeito.

Embora existam outras escalas desenvolvidas para estimar o risco de reações adversas anticolinérgicas, tais como Drug Burden Index (DBI-Ach) (HILMER et al., 2007), Anticholinergic Drug Scale (ADS) (CARNAHAN et al., 2006) e Anticholinergic Risk Scale (ARS) (RUDOLPH et al., 2008), a ACB foi elaborada com foco nos efeitos adversos cognitivos desses fármacos. Diante da crescente prevalência de déficit cognitivo em idosos e da inquestionável importância de utilizar estratégias que possam ajudar a evitar que efeitos adversos sejam confundidos com sintomas de enfermidades, o emprego da ACB na prática clínica pode ser de grande valia.

Não há relatos científicos sobre o uso dessa escala no Brasil. Por outro lado, a utilidade e a eficácia da escala na identificação e na prevenção de efeitos adversos associados a certos fármacos precisam ser amplamente investigadas, especialmente em estudos clínicos (BOUSTANI et al., 2008). Por conseguinte, o objetivo da presente revisão foi reunir os achados obtidos em estudos clínicos que investigaram a associação entre alterações cognitivas e a escala ACB.

\section{Métodos}

Para a obtenção dos artigos utilizados neste trabalho, realizou-se uma revisão integrativa da literatura. A busca de artigos foi conduzida em maio, 
junho e julho de 2015. As bases de dados utilizadas foram a US National Library of Medicine National Institutes of Health (MEDLINE/PubMed) e a Biblioteca Virtual de Saúde (BVS-Bireme).

Como esta investigação se limitava especificamente aos estudos em que foi aplicada a escala ACB, o termo utilizado na busca foi "anticholinergic cognitive burden". A leitura e a seleção dos artigos foram norteadas pela seguinte questão: nos estudos clínicos que investigaram a associação entre alterações cognitivas e ACB, quais os achados obtidos sobre essa associação? Portanto, foram incluídos nesta revisão apenas artigos originais completos sobre estudos clínicos cujos achados constituíam resposta para a questão norteadora.

Por conseguinte, atenderam aos critérios de inclusão os estudos clínicos que investigaram a associação entre ACB e alterações cognitivas por meio de um ou mais instrumentos de avaliação cognitiva. Foram excluídos os estudos cujos idiomas não eram inglês, português ou espanhol e os artigos incompletos (resumos).

A Figura 1 apresenta o fluxograma da seleção da amostra para revisão. A busca inicial nas bases de dados gerou 36 artigos completos. Após a exclusão dos repetidos (duplicados nas bases de dados utilizadas) e daqueles cujos idiomas não eram inglês, português ou espanhol, restaram 25 artigos. Com a seleção dos estudos clínicos, restaram 11 artigos que respondiam à questão norteadora. 


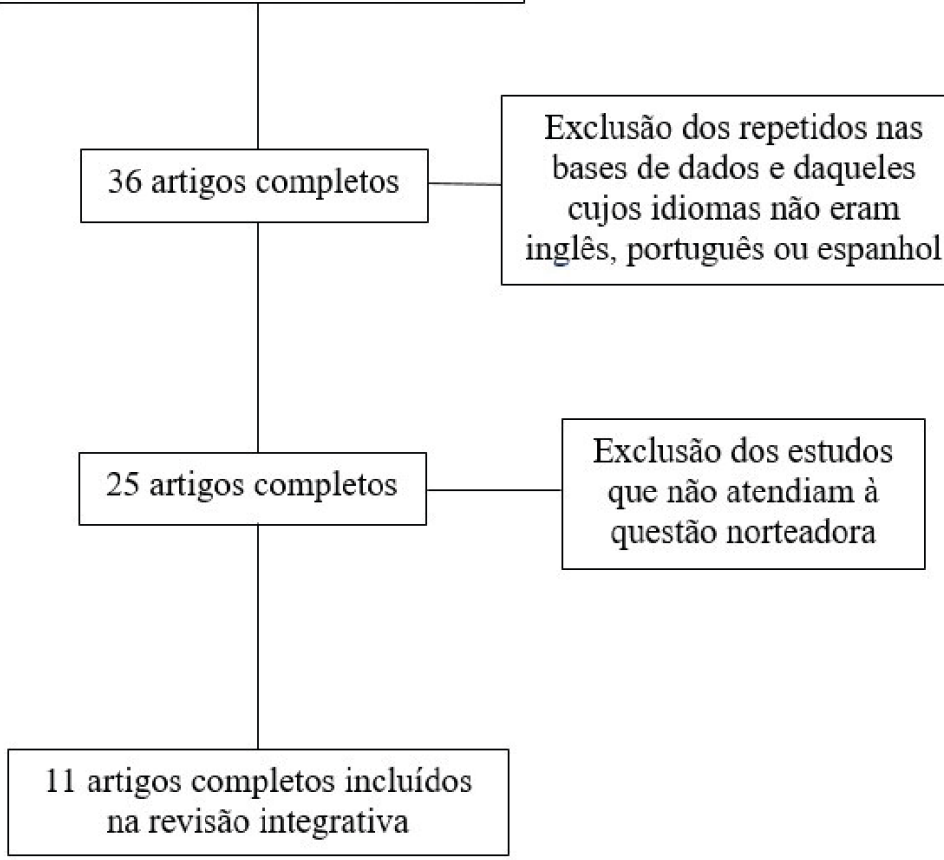

E
L
$\mathrm{E}$
$\mathrm{G}$
$\mathrm{I}$
$\mathrm{B}$
$\mathrm{I}$
$\mathrm{L}$
$\mathrm{I}$
$\mathrm{D}$
$\mathrm{A}$
$\mathrm{D}$
$\mathrm{E}$

$\mathrm{I}$
$\mathrm{N}$
$\mathrm{C}$
$\mathrm{L}$
$\mathrm{U}$
$\mathrm{S}$
$\widetilde{A}$
$\mathrm{O}$

Exclusão dos estudos que nào atendiam à questão norteadora

\section{Fonte: Figura elaborado pela autora.}

Como a publicação da escala ocorreu em 2008, o artigo mais antigo que se enquadrava nos critérios desta revisão foi publicado em 2009. Portanto, foram incluídos artigos publicados de 2009 até julho de 2015.

Após a leitura, na íntegra, dos 11 artigos encontrados, foram extraídas as seguintes informações: país onde o estudo foi realizado, tipo (delineamento) de pesquisa, amostra (sujeitos), instrumentos de avaliação cognitiva utilizados e achados obtidos por meio da aplicação desses instrumentos. A identificação do instrumento de avaliação cognitiva utilizado foi de grande relevância porque vários estudos utilizaram a escala $\mathrm{ACB}$ apenas para quantificar a carga 
anticolinérgica da farmacoterapia dos sujeitos da pesquisa sem, no entanto, aplicar algum instrumento de avaliação cognitiva.

\section{Resultados}

A Tabela 1 resume as características (país, tipo de pesquisa, amostra, instrumentos de avaliação cognitiva aplicados) e os achados obtidos pelos 11 estudos que utilizaram a escala ACB. Todos os estudos selecionados foram publicados em periódicos indexados no MEDLINE. A maior parte deles $(\mathrm{n}=8)$ foi conduzida nos Estados Unidos. Com exceção de um estudo que incluiu 1.304 indivíduos com idade maior ou igual $\geq 55$ (média de idade $=81$ anos), todos os demais artigos se referiam a sujeitos com mais de 60 anos.

Tabela 1 - Artigos selecionados, segundo autores, país, tipo de estudo, amostra, instrumentos de avaliação cognitiva utilizados e achados obtidos por meio desses instrumentos.

\begin{tabular}{|c|c|c|c|c|c|}
\hline Referência & País & $\begin{array}{l}\text { Tipo de } \\
\text { Estudo }\end{array}$ & $\begin{array}{l}\text { Amostra } \\
\text { (sujeitos) }\end{array}$ & Instrumentos & Achados obtidos \\
\hline $\begin{array}{l}\text { Block } \\
\text { et al., } \\
2015\end{array}$ & EUA & Transversal & $\begin{array}{l}290 \text { de ambos } \\
\text { os sexos com } \\
\text { idade } \geq 65 \\
\text { (média }=73 \pm 5 \text { ) }\end{array}$ & RBANS & $\begin{array}{c}\text { Maior ACB associada } \\
\text { com pior performance } \\
\text { no RBANS. }\end{array}$ \\
\hline $\begin{array}{l}\text { Kashyap } \\
\text { et al., } \\
2014\end{array}$ & Canadá & $\begin{array}{l}\text { Longitudi- } \\
\text { nal (segui- } \\
\text { mento de } \\
1 \text { ano) }\end{array}$ & $\begin{array}{l}102 \text { de ambos } \\
\text { os sexos com } \\
\text { idade } \geq 60 \\
\text { (média }=72 \pm 7 \text { ) }\end{array}$ & $\begin{array}{c}\text { MMSE, } \\
\text { MDRS, } \\
\text { MOCA, RL/ } \\
\text { RI, ROCFT, } \\
\text { WAIS, TMT, } \\
\text { VST, QAM, } \\
\text { DSM-V }\end{array}$ & $\begin{array}{c}\text { Maior ACB associada } \\
\text { com comprometi- } \\
\text { mento da memória. }\end{array}$ \\
\hline $\begin{array}{l}\text { Koyama } \\
\text { et al., } \\
2014\end{array}$ & EUA & $\begin{array}{l}\text { Longitudi- } \\
\text { nal (segui- } \\
\text { mento de } \\
5 \text { anos) }\end{array}$ & $\begin{array}{l}1.429 \text { mulheres } \\
\text { com idade } \geq \\
75 \text { (média }= \\
83 \pm 3 \text { na linha } \\
\text { de base) }\end{array}$ & $\begin{array}{c}\text { Trails B, } \\
\text { 3MS, CVLT-II, } \\
\text { BDSPAN e } \\
\text { testes de } \\
\text { memória } \\
\text { semântica }\end{array}$ & $\begin{array}{c}\text { Maior ACB associada } \\
\text { com pior performance } \\
\text { cognitiva. }\end{array}$ \\
\hline $\begin{array}{l}\text { Koyama } \\
\text { et al., } \\
2013\end{array}$ & EUA & $\begin{array}{l}\text { Longitudi- } \\
\text { nal (segui- } \\
\text { mento de } \\
10 \text { anos) }\end{array}$ & $\begin{array}{c}1.484 \text { mulheres } \\
\text { com idade } \geq \\
75 \text { (média }= \\
78 \pm 3 \text { na linha } \\
\text { de base) }\end{array}$ & MMSE & $\begin{array}{l}\text { Maior ACB associada } \\
\text { com função cognitiva } \\
\text { mais comprometida. }\end{array}$ \\
\hline
\end{tabular}


continuação

\begin{tabular}{|c|c|c|c|c|c|}
\hline Referência & País & $\begin{array}{l}\text { Tipo de } \\
\text { Estudo }\end{array}$ & $\begin{array}{l}\text { Amostra } \\
\text { (sujeitos) }\end{array}$ & Instrumentos & Achados obtidos \\
\hline $\begin{array}{l}\text { Pasina } \\
\text { et al., } \\
2013\end{array}$ & Itália & $\begin{array}{l}\text { Transversal } \\
\text { (segui- } \\
\text { mento de } \\
3 \text { meses) }\end{array}$ & $\begin{array}{c}1.380 \text { de } \\
\text { ambos os } \\
\text { sexos com } \\
\text { idade } \geq 65 \\
\text { (média }=79 \pm 7 \text { ) }\end{array}$ & SBT & $\begin{array}{l}\text { Maior ACB associada } \\
\text { com função cognitiva } \\
\text { mais comprometida. }\end{array}$ \\
\hline $\begin{array}{l}\text { Shah } \\
\text { et al., } \\
2013\end{array}$ & EUA & $\begin{array}{l}\text { Longitudi- } \\
\text { nal (segui- } \\
\text { mento de } \\
10 \text { anos) }\end{array}$ & $\begin{array}{c}896 \text { com idade } \\
\text { média }=76 \pm 6 \\
\text { na linha de } \\
\text { base) }\end{array}$ & $\begin{array}{l}\text { MMSE e uma } \\
\text { bateria de } 19 \\
\text { testes cogni- } \\
\text { tivos descrita } \\
\text { por Wilson et } \\
\text { al. (2005) }\end{array}$ & $\begin{array}{l}\text { Idosos que iniciaram } \\
\text { tratamento com anti- } \\
\text { colinérgicos tiveram } \\
\text { um declínio cognitivo } \\
\text { mais acentuado } \\
\text { do que os que não } \\
\text { utilizam. }\end{array}$ \\
\hline $\begin{array}{l}\text { Cai } \\
\text { et al., } \\
2013\end{array}$ & EUA & $\begin{array}{l}\text { Longitudi- } \\
\text { nal (segui- } \\
\text { mento de } \\
1 \text { ano) }\end{array}$ & $\begin{array}{l}3.690 \text { de } \\
\text { ambos os } \\
\text { sexos com } \\
\text { idade } \geq 65\end{array}$ & $\begin{array}{l}\text { 6CIT e } \\
\text { CSI-D }\end{array}$ & $\begin{array}{l}\text { Elevada ACB foi } \\
\text { associada com risco } \\
\text { de déficit cognitivo } \\
\text { após } 2 \text { a } 3 \text { meses de } \\
\text { exposição contínua. }\end{array}$ \\
\hline $\begin{array}{l}\text { Fox } \\
\text { et al., } \\
2011\end{array}$ & Inglaterra & $\begin{array}{l}\text { Longitudi- } \\
\text { nal (segui- } \\
\text { mento de } \\
1 \text { ano e } \\
\text { meio) }\end{array}$ & $\begin{array}{c}1.304 \text { de } \\
\text { ambos os } \\
\text { sexos com } \\
\text { idade } \geq 55 \\
\text { (média }=75 \pm 7 \\
\text { na linha de } \\
\text { base) }\end{array}$ & MMSE & $\begin{array}{l}\text { O uso de anticolinér- } \\
\text { gicos com escore } 2 \\
\text { e } 3 \text { na escala ACB foi } \\
\text { associado, de modo } \\
\text { dose-dependente, } \\
\text { com pior desempe- } \\
\text { nho no MMSE. }\end{array}$ \\
\hline $\begin{array}{l}\text { Campbell } \\
\text { et al., } \\
2011\end{array}$ & EUA & $\begin{array}{l}\text { Observa- } \\
\text { cional }\end{array}$ & $\begin{array}{c}147 \text { de ambos } \\
\text { os sexos } \\
\text { com idade } \\
\geq 65 \text { (média } \\
=77 \text { anos), } \\
\text { hospitalizados } \\
\text { e com déficit } \\
\text { cognitivo. }\end{array}$ & CAM & $\begin{array}{l}\text { Os resultados não } \\
\text { confirmaram a asso- } \\
\text { ciação entre o uso } \\
\text { de anticolinérgicos e } \\
\text { delírio em idosos com } \\
\text { déficit cognitivo. }\end{array}$ \\
\hline $\begin{array}{l}\text { Campbell } \\
\text { et al., } \\
2010\end{array}$ & $\begin{array}{l}\text { EUA e } \\
\text { Nigéria }\end{array}$ & $\begin{array}{l}\text { Longitudi- } \\
\text { nal (segui- } \\
\text { mento de } \\
6 \text { anos) }\end{array}$ & $\begin{array}{c}1.652 \text { de } \\
\text { ambos os } \\
\text { sexos com } \\
\text { idade } \geq 65 \\
\text { (média }=82 \pm 5 \\
\text { na linha de } \\
\text { base) }\end{array}$ & $\begin{array}{c}\text { CSI-D, } \\
\text { CERAD, CHIF } \\
\text { e CAMDEX }\end{array}$ & $\begin{array}{l}\text { O uso de anticolinérgi- } \\
\text { cos com escore } 2 \text { e } 3 \\
\text { na escala ACB elevou } \\
\text { o risco de déficit } \\
\text { cognitivo. }\end{array}$ \\
\hline $\begin{array}{l}\text { Kolanowski } \\
\text { et al., } \\
2009\end{array}$ & EUA & Transversal & $\begin{array}{l}87 \text { idosos de } \\
\text { ambos os } \\
\text { sexos com } \\
\text { idade } \geq 65 \\
\text { (média }=86 \pm 6 \text { ) }\end{array}$ & MMSE & $\begin{array}{l}\text { Os resultados do } \\
\text { MMSE não apontaram } \\
\text { diferença entre os ido- } \\
\text { sos que utilizavam e } \\
\text { os que não utilizavam } \\
\text { anticolinérgicos. }\end{array}$ \\
\hline
\end{tabular}

RBANS = Assessment of Neuropsychological Status; MMSE = Mini-Exame do Estado Mental;

MDRS = Mattis Dementia Rating Scale; MoCA = Montreal Cognitive Assessment; RL/Rl = Free and 
Cued Recall; ROCFT = Rey-Osterrieth Complex Figure Test; WAIS = Wechsler Adult Intelligence Scale; TMT = Trail Making Test; VST = Victoria Stroop Test; QAM - Questionnaire d'auto-évaluation de la mémoire; DSM-V = Diagnostic and Statistical Manual of Mental Disorders, Fifth Edition; 3MS = Modified Mini Mental State Examination; CVLT-II = California Verbal Learning Test, Second Edition; BDSPAN = Backward Digit Span; SBT = Short Blessed Test; 6CIT = 6 Item Cognitive Impairment Test (Callahan et al., 2002); CSI-D = Community Screening Instrument for Dementia; CAM = Confusion Assessment Method; CERAD = Consortium to Establish a Registry for Alzheimer's Disease, $\mathrm{CHIF}=$ The Clinician Home-Based Interview to assess Function; CAMDEX = Cambridge Examination for Mental Disorders of the Elderly.

Fonte: Tabela elaborada pela autora.

Quanto ao delineamento, compuseram a amostra sete estudos longitudinais, três transversais e um observacional. Entre os eventos adversos cognitivos que são previstos pela escala ACB (declínio cognitivo, demência e delírio), apenas um dos artigos estudados investigou a ocorrência de delírio. Dos 10 restantes, que buscaram associar déficit cognitivo e a escala ACB, apenas um não observou diferença entre os idosos que utilizavam e os que não utilizavam fármacos com atividade anticolinérgica. Este foi também o estudo com a menor amostra (87 idosos).

Em todos os estudos incluídos nesta revisão, o estado cognitivo dos sujeitos foi avaliado por meio de instrumentos apropriados. A inclusão dessa avaliação foi critério para a seleção da amostra desta revisão. Dos dez estudos que avaliaram a relação entre estado cognitivo e ACB, nove observaram associação desta última com pior performance em testes de cognição. Embora, como mostram os resultados, essa associação tenha sido demonstrada por meio da aplicação de uma grande variedade de testes de avaliação cognitiva, o MMSE foi o instrumento mais empregado (Tabela 1).

Com base nos artigos analisados, em geral, os efeitos adversos cognitivos ocorrem em decorrência da exposição a pelo menos um fármaco com atividade anticolinérgica moderada ou severa (escores 2 e 3 ) ou cumulativamente, como resultado do uso concomitante de vários fármacos, cuja somatória configure alta carga anticolinérgica. O risco de efeitos adversos é, portanto, dose-dependente e parece ser maior com o uso contínuo.

\section{Discussão}

Medicamentos são recursos essenciais que previnem e curam doenças, aliviam sintomas e melhoram a qualidade de vida. No entanto, com o envelhecimento, o organismo sofre alterações que o tornam mais sujeito aos efeitos tóxicos e/ou à ineficácia dos fármacos (BORJA-OLIVEIRA, 2010). Ainda que a escala ACB tenha sido criteriosamente elaborada para identificar e prevenir, 
em idosos, efeitos adversos cognitivos de fármacos com atividade anticolinérgica, a avaliação da sua utilidade e eficácia deve ser investigada clinicamente (BOUSTANI et al., 2008).

A escala ACB foi desenvolvida com o propósito de reduzir a carga anticolinérgica em idosos, uma vez essa faixa etária é mais exposta e particularmente vulnerável aos efeitos desses fármacos (BOUSTANI et al., 2008). Realmente, os sujeitos dos estudos clínicos que empregaram a ACB eram idosos. Esses indivíduos estão mais sujeitos à toxicidade dos efeitos adversos anticolinérgicos no SNC. Esta maior sensibilidade está associada à significativa diminuição de neurônios e receptores colinérgicos funcionais, ao aumento da permeabilidade da barreira hematoencefálica e à redução no metabolismo hepático e na excreção renal (TUNE, 2001; CAMPBELL et al., 2009).

Tal como demonstrado nesta revisão, estudos clínicos têm, de fato, evidenciado e estabelecido a relação entre $\mathrm{ACB}$ e déficit cognitivo. Nove dos dez estudos que avaliaram a relação entre estado cognitivo e $\mathrm{ACB}$, observaram associação desta última com pior performance nos testes de cognição. Essa associação foi demonstrada por meio da aplicação de uma grande variedade de testes de avaliação cognitiva. Embora o MMSE tenha sido o instrumento mais empregado, em metade desses estudos os autores utilizaram mais de um instrumento.

Kashyap et al. (2014) demonstraram que, em estudos sobre carga anticolinérgica, os resultados da avaliação cognitiva podem diferir para um mesmo individuo conforme o teste aplicado. Os métodos utilizados para medir a alteração cognitiva decorrente da carga anticolinérgica podem ter efeito significativo sobre os resultados. Para esses autores (KASHYAP et al., 2014), realizar uma análise de sensibilidade usando dois ou mais métodos pode ser uma maneira de aumentar a confiança nas conclusões. Os pesquisadores devem estar cientes dessas diferenças e selecionar instrumentos de estudo e métodos analíticos que melhor se ajustem aos objetivos de seu estudo (KASHYAP et al., 2014).

Nos artigos existentes sobre os efeitos adversos cognitivos dos anticolinérgicos, é frequente a referência à possibilidade de que os mesmos sejam confundidos com sintomas de demência. Observa-se que essa é uma das principais justificativas que mobilizam os autores desses trabalhos. Muitos deles mencionam a possibilidade de erro no diagnóstico de demência, incluindo a precoce, quando na verdade os pacientes estão experimentando declínio cognitivo secundário ao uso de fármacos anticolinérgicos (CARRIÈRE et al., 2009; CAMPBELL et al., 2010).

O artigo de Rigo et al. (2006) ilustra claramente de que modo um déficit cognitivo decorrente de efeito adverso de tratamento farmacológico pode ser confundido com doença mental. Os autores relataram o caso de uma octogenária 
portadora de tremor essencial, que foi confundido com doença de Parkinson e tratado com biperideno. Esse fármaco anticolinérgico, em doses elevadas, causou importante déficit cognitivo, quedas frequentes, fratura do quadril, perda da autonomia física e psíquica e consequente institucionalização em lar geriátrico. $\mathrm{O}$ déficit cognitivo causado pelo biperideno foi ainda erroneamente diagnosticado como demência de Alzheimer, configurando a cascata iatrogênica. Há relatos de que é uma situação relativamente frequente o atendimento a pacientes idosos utilizando anticolinérgicos para tratamento de tremor ou síndrome parkinsoniana que desenvolvem sintomas demenciais que se revertem com a suspensão do fármaco (NITRINI, 2006).

De fato, o caráter reversível do déficit cognitivo produzido pelos anticolinérgicos tem sido demonstrado. Existem relatos de efeitos adversos cognitivos que ocorrem durante o uso contínuo e se revertem após a descontinuação (CARRIÈRE et al., 2009). No caso da octogenária, mencionado acima, a suspensão do biperideno resultou em reversão completa do quadro demencial e resgate da independência da idosa (RIGO et al., 2006). É provável que essa reversão dependa da farmacocinética (clearance) e da farmacodinâmica (dissociação de receptores muscarínicos no cérebro) (KASHYAP et al., 2014).

Apesar da conhecida associação entre delírio e uso de anticolinérgicos (CAMPBELL et al., 2009; AGOSTINI; LEO-SUMMERS; INOUYE, 2001; CAEIRO et al., 2004), o único estudo (CAMPBELL et al., 2011) sobre incidência de delírio que se enquadrou nos objetivos da presente revisão não confirmou essa relação. Nesse estudo, a prescrição de medicamentos anticolinérgicos para idosos hospitalizados com comprometimento cognitivo não aumentou a incidência de delírio. Para explicar o resultado considerado inesperado, os autores especularam o fato de que os resultados se basearam apenas no registro de prescrição, sem que houvesse confirmação de que os idosos realmente receberam os medicamentos. Consideraram também a falta de informação sobre quais desses medicamentos eram novas prescrições ou prescrições anteriores de uso contínuo, bem como a possível subnotificação de episódios de delírio. Lembraram ainda que os estudos anteriores que confirmaram a relação entre anticolinérgicos e delírio mediram a prevalência e não a incidência de delírio.

De qualquer maneira, as evidências encontradas na literatura científica mostram a importância da revisão da farmacoterapia de todo paciente que apresentar sinais iniciais ou crônicos de déficit cognitivo ou de delírio. Essa revisão deve incluir também os medicamentos isentos de prescrição e a automedicação. O emprego da ACB pode ser útil para auxiliar tanto para o reconhecimento dos medicamentos com atividade anticolinérgica em uso como para estimar a carga anticolinérgica à qual o paciente está sujeito. Essa 
avaliação se dá com base no escore individual de cada medicamento em uso e na somatória dos escores daqueles usados concomitantemente. A identificação de um medicamento com uma pontuação 2 ou 3 na escala ACB ou de medicamentos usados concomitantemente cuja soma dos escores resulte em uma pontuação acima de 3 requer reavaliar a continuidade do uso e considerar a possibilidade de substituição por fármaco(s) alternativo(s), com menor (ou sem) atividade anticolinérgica (BOUSTANI et al, 2008). Afinal, o comprometimento dos anticolinérgicos sobre a performance cognitiva e física pode ocorrer em resultado do efeito cumulativo do uso concomitante de medicamentos com atividade muscarínica modesta (PASINA et al., 2013). Evidentemente, a retirada de fármacos deve ser feita de maneira adequada e, quando necessário, gradual para evitar eventos indesejáveis, como por exemplo, efeito rebote (BOUSTANI et al., 2008).

O tratamento de pessoas idosas com fármacos dotados de atividade anticolinérgica deve ser, portanto, criteriosamente avaliado (SHAH et al., 2013). É essencial evitar o uso de anticolinérgicos em idosos, considerar os riscos, os benefícios e os eventuais danos associados a esses medicamentos (CAI et al., 2013; CAMPBELL et al., 2010), especialmente quando há possibilidade de que o tratamento dure mais do que 2 ou 3 meses (CAI et al., 2013).

Com a crescente prevalência de déficit cognitivo em idosos, os prescritores devem também estar cientes do impacto dos anticolinérgicos no possível agravo dos sintomas (FOX et al., 2011; CAMPBELL et al., 2010). Um estudo com 3.251 idosos institucionalizados mostrou que quase metade deles utilizava agentes colinérgicos (anticolinesterásicos) concomitantemente com anticolinérgicos. Estes últimos antagonizam os anticolinesterásicos (donepezila, rivastigmina e galantamina), empregados no tratamento da demência (MODI et al., 2009).

A escala ACB é também utilizada como referência para atividade adversa anticolinérgica na seleção americana de fármacos potencialmente inapropriados para idosos, conhecida como critérios de Beers (AGS, 2012). Subsequentemente, é referência no consenso brasileiro sobre medicamentos inapropriados (OLIVEIRA et al., 2015; 2016). Afinal, este (o consenso brasileiro) foi elaborado a partir da lista americana, embora tenha sido desenvolvido com base na opinião (método Delphi) de especialistas brasileiros. Há estudos no Brasil que empregaram como ferramenta os critérios de Beers. Nesses estudos, portanto, a $A C B$, ainda que não seja mencionada, é referência para atividade adversa anticolinérgica, mesmo que implicitamente.

Os pacientes idosos frequentemente apresentam sinais e sintomas de declínio cognitivo e deterioração funcional. Reconhecer e tratar potenciais causas e contribuintes para o declínio cognitivo e funcional é fundamental se 
quisermos evitar a institucionalização desnecessária e melhorar a qualidade de vida. Promover a saúde, a autonomia e a independência de pacientes idosos é essencial para que possam viver em seus domicílios o maior tempo possível. Em particular, os idosos são mais propensos do que os pacientes mais jovens a desenvolver comprometimento cognitivo ou funcional como resultado do uso de medicamentos. Ao avaliar pacientes idosos que apresentem esses sinais, é necessária uma avaliação completa sobre diagnósticos e medicamentos em uso. É importante não assumir que o declínio cognitivo é demência "until you have turned over all the rocks" (FARRELL; EISENER-PARSCHE; DALTON, 2014), ou numa versão em português, até que todas as outras possíveis causas tenham sido descartadas. Esse precisa ser um dos nossos compromissos. Se o envelhecimento populacional e o aumento da expectativa de vida conduzem ao aumento da utilização de serviços de saúde e de medicamentos, cada vez mais teremos que confrontar esses desafios (BORJA-OLIVEIRA, 2013).

\section{Considerações finais}

O uso disseminado e banal de medicamentos, especialmente na velhice, representa uma oposição ao princípio hipocrático "primum non nocere" (primeiro, não causar dano). Os eventos adversos discutidos nesta revisão são evitáveis, não apenas pela prescrição e dispensação racional mas também pela revisão constante da farmacoterapia individual. Nessa avaliação, deve-se considerar a carga anticolinérgica, o uso de medicamentos inapropriados para idosos, as doses utilizadas, a presença de eventuais interações medicamentosas e de efeitos adversos.

Deve-se ainda investigar se há possibilidade de que o indivíduo esteja utilizando medicamentos para tratar efeitos adversos de outros, configurando o que chamamos de cascata iatrogênica. $O$ uso de fármacos para tratar ou prevenir efeitos adversos de outros é muitas vezes racional (por exemplo, o emprego de gastroprotetor para evitar efeitos adversos gástricos de certos tratamentos farmacológicos em certas circunstâncias e por tempo determinado). Todavia, a literatura científica relata abundantemente, nos mais diversos cenários, que efeitos adversos nem sempre são reconhecidos, seja por usuários seja por profissionais. Nesta revisão sobre os efeitos adversos cognitivos dos anticolinérgicos, observou-se que é frequente por parte dos autores a referência à possibilidade de que tais efeitos adversos sejam confundidos com sintomas de demência. É notório que essa é uma das principais razões que mobilizam os autores desses estudos. 
Muitos anticolinérgicos são isentos de prescrição ou vendidos sem que a prescrição médica seja exigida, fato que demanda intervenções educativas no sentido de alertar a população sobre os riscos da automedicação. O idoso, comumente polimedicado, deve ser alvo estratégico dessas intervenções, sobretudo diante do envelhecimento populacional no qual o Brasil é destaque. Por outro lado, é também estratégica e essencial a comunicação clara entre paciente e profissional sobre os possíveis efeitos adversos do seu tratamento, de modo que ele possa também reconhecê-los como eventos adversos antes de interpretá-los como doença.

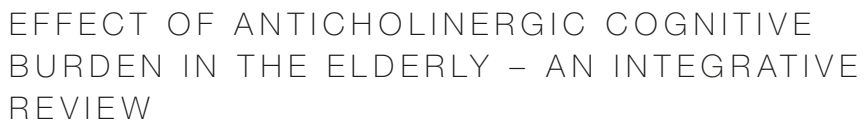

Elderly people are more susceptible to cognitive adverse effects of medications with anticholinergic activity. This is due to factors associated with aging, such as decline in central cholinergic activity, increase in blood-brain barrier permeability, decline in hepatic and renal metabolism and polypharmacy. The Anticholinergic Cognitive Burden (ACB) scale was developed reduce the risk for developing adverse cognitive outcomes such as cognitive impairment, dementia and delirium. The purpose of this integrative review is to bring together findings from clinical studies on the association between cognitive impairment and ACB scale. The search in MEDLINE/PubMed and BVS-Medicine bases generated 11 full articles in English. Seven were longitudinal, three transversal and one observational. Eight of them were conducted in the United States. Nine of 10 showed the relationship between the ACB and cognitive impairment due to at least one moderate or severe anticholinergic medication or due to concomitant use of multiple medications. MMSE was the most commonly cognitive assessment. Evidence shows the importance of the review of pharmacotherapy of all patients with cognitive impairment or delirium. It is important not to assume that cognitive decline is dementia until pharmacologic causes have been excluded. 

Elderly.

\author{
referências
}

AGOSTINI, Joseph V.; Leo-Summers, Linda S.; Inouye, Sharon K. Cognitive and other adverse effects of diphenhydramine use in hospitalized older adults. Archives of Internal Medicine, Chicago, v. 161, n. 17, p. 2091-2097, set. 2001.

ALBUQUERQUE, Edson X. et al. Mammalian nicotinic acetylcholine receptors: from structure to function. Physiological Reviews, Bethesda, v. 89, n. 1, p. 73-120, jul. 2009.

AMERICAN GERIATRICS SOCIETY (AGS). American Geriatrics Society updated Beers Criteria for potentially inappropriate medication use in older adults. Journal of American Geriatrics Society, New York, v. 60, n. 4, p. 616-631, abr. 2012.

BLOCK, Cady K. et al. The interaction between medical burden and anticholinergic cognitive burden on neuropsychological function in a geriatric primary care sample. Archives of Clinical Neuropsychology, New York, v. 30, n. 2, p. 105-113, mar. 2015.

BORJA-OLIVEIRA, Caroline R. Atenção ao idoso no domicílio: o enfoque da farmácia. In: DOMINGUES, Marisa A.; LEMOS, Naira R. (Org.). Gerontologia: os desafios nos diversos cenários de atenção. São Paulo: Manole, 2010. p. 355-366.

BORJA-OLIVEIRA, Caroline R. Organizadores e cortadores de comprimidos: riscos e restrições ao uso. Revista de Saúde Pública, São Paulo, v. 47, n. 1, p. 123-127, fev. 2013.

Boustani, Malaz A. et al. Impact of anticholinergics on the aging brain: a review and practical application. Aging Health, London, v. 4, n. 3, p. 311-320, 2008.

CAl, Xueya et al. Long-term anticholinergic use and the aging brain. Alzheimer's \& Dementia, Orlando, v. 9, n. 4, p. 377-385, jul. 2013.

CAEIRO, Lara et al. Delirium in acute stroke: a preliminary study of the role of anticholinergic medications. European Journal of Neurology, Oxford, v. 11, n. 10, p. 699-704, out. 2004.

CAMPBELL, Noll et al. Association between prescribing of anticholinergic medications and incident delirium: a cohort study. Journal of American Geriatrics Society, New York, v. 59, supl. 2, p. 277-281, nov. 2011

CAMPBELL, Noll et al. The cognitive impact of anticholinergics: a clinical review. Clinical Interventions in Aging, Auckland, v. 4, p. 225-233, jun. 2009.

CAMPBELL, Noll et al. Use of anticholinergics and the risk of cognitive impairment in an African American population. Neurology, New York, v. 75, n. 2, p. 152-159, jul. 2010.

CARNAHAN, Ryan M. et al. The Anticholinergic Drug Scale as a measure of drug-related anticholinergic burden: Associations with serum anticholinergic activity. Journal of Clinical Pharmacology, Stamford, v. 46, n. 12, p. 1481-1486, dez. 2006.

CARRIËRE, Isabelle et al. Drugs with anticholinergic properties, cognitive decline, and dementia in an elderly general population: the 3-city study. Archives of Internal Medicine, Chicago, v. 169, n. 14, p. 1317-1324, jul. 2009.

FARRALL, Andrew J.; Wardlaw, Joanna M. Blood-brain barrier: ageing and microvascular disease: systematic review and meta-analysis. Neurobiology of Aging, New York, v. 30, n. 3, p. 337-352, mar. 2009 
FARRELL, Barbara; Eisener-Parsche, Pamela; Dalton, Dan. Turning over the rocks: role of anticholinergics and benzodiazepines in cognitive decline and falls. Canadian Family Physician, Mississauga, ON, v. 60, n. 2, p. 345-350, abr. 2014.

FOX, Chris et al. Anticholinergic Medication Use and Cognitive Impairment in the Older Population: The Medical Research Council Cognitive Function and Ageing Study. Journal of American Geriatrics Society, New York, v. 59, n. 8, p. 1477-1483, ago. 2011.

HILMER, Sarah N. et al. A drug burden index to define the functional burden of medications in older people. Archives of Internal Medicine, Chicago, v. 167, n. 8, p. 781-787, abr. 2007.

KASHYAP, Mandavi et al. Methodological Challenges in Determining Longitudinal Associations Between Anticholinergic Drug Use and Incident Cognitive. Journal of American Geriatrics Society, New York, v. 62, n. 2, p. 336-341, jan. 2014.

KOLANOWSKI, Ann et al. A Preliminary Study of Anticholinergic Burden and Relationship to a Quality of Life Indicator, Engagement in Activities, in Nursing home Residents with Dementia. Journal of the American Medical Directors Association, Hagerstown, v. 10, n. 4, p. 252-257, maio 2009.

KOYAMA, Alain et al. Long-term Cognitive and Functional Effects of Potentially Inappropriate Medications in Older Women. Journals of Gerontology: Series A, Washington, D.C., v. 69, n. 4, p. 423-429, abr. 2014.

KOYAMA, Alain et al. Ten-Year Trajectory of Potentially Inappropriate Medications in Very Old Women: Importance of Cognitive Status. Journal of American Geriatrics Society, New York, v. 61, n. 2, p. 258-263, fev. 2013.

MODI, Ankita et al. Concomitant use of anticholinergics with acetylcholinesterase inhibitors in Medicaid recipients with dementia and residing in nursing homes. Journal of American Geriatrics Society, New York, v. 57, n. 7, p. 1238-1244, abr. 2009.

NITRINI, Ricardo. Há sentido em utilizar o tratamento atualmente disponível para a doença de Alzheimer? Revista de Psiquiatria Clínica, São Paulo, v. 33, n. 4, p. 214-217, 2006.

OLIVEIRA, Márcio G. et al. Brazilian consensus of potentially inappropriate medications in the elderly: preliminary data. Journal of American Geriatrics Society, New York, v. 63, supl. 1, p. A147, abr. 2015

OLIVEIRA, Márcio G. et al. Consenso Brasileiro de Medicamentos Potencialmente Inapropriados para Idosos. Geriatrics, Gerontolology and Aging, Rio de Janeiro, v. 10, n. 4, p. 168-181, jun. 2016.

PASINA, Luca et al. Association of anticholinergic burden with cognitive and functional status in a cohort of hospitalized elderly: comparison of the anticholinergic cognitive burden scale and anticholinergic risk scale: results from the REPOSI study. Drugs \& Aging, Auckland, v. 30, n. 2, p. 103-112, fev. 2013.

POPESCU, Bogdan O. et al. Blood-brain barrier alterations in ageing and dementia. Journal of Neurological Sciences, Amsterdam, v. 283, n. 1-2, p. 99-106, ago. 2009.

RIGO, Julio C. et al. Demência reversível e quedas associadas ao biperideno. Revista de Psiquiatria Clínica, São Paulo, v. 33, n. 1, p. 24-27, 2006.

RUDOLPH, James L. et al. The Anticholinergic Risk Scale and anticholinergic adverse effects in older persons. Archives of Internal Medicine, Chicago, v. 168, n. 5, p. 508-513, mar. 2008.

SHAH, Raj C. et al. Cognitive Decline in Older Persons Initiating Anticholinergic Medications. PLoS One, San Francisco, v. 8, n. 5, maio 2013.

TUNE, Larry E. Anticholinergic effects of medication in elderly patients. The Journal of Clinical Psychiatry, Memphis, v. 62, supl. 21, p. 11-14, 2001. 
VENTURA, Ana L. M. et al. Sistema colinérgico: revisitando receptores, regulação e a relação com a doença de Alzheimer, esquizofrenia, epilepsia e tabagismo. Revista de Psiquiatria Clínica, São Paulo, v. 37, n. 2, p. 66-72, 2010.

WILSON, Robert S. et al. Early and late life cognitive activity and cognitive systems in old age. Journal of the International Neuropsychological Society, Cambridge, v. 11, n. 4, p. 400-407, jul. 2005.

Data de submissão: 24/11/2015

Data de aprovação: 23/10/2017 
Escala de Carga Anticolinérgica Cognitiva (ACB).

\begin{tabular}{|c|c|c|c|}
\hline & Atividade Possível & Atividade $\mathrm{De}$ & inida \\
\hline & Escore 1 & Escore 2 & Escore 3 \\
\hline 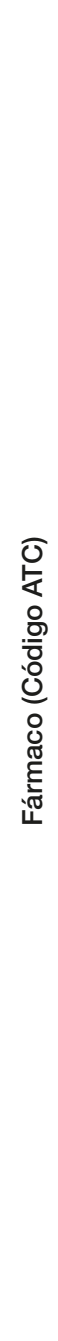 & 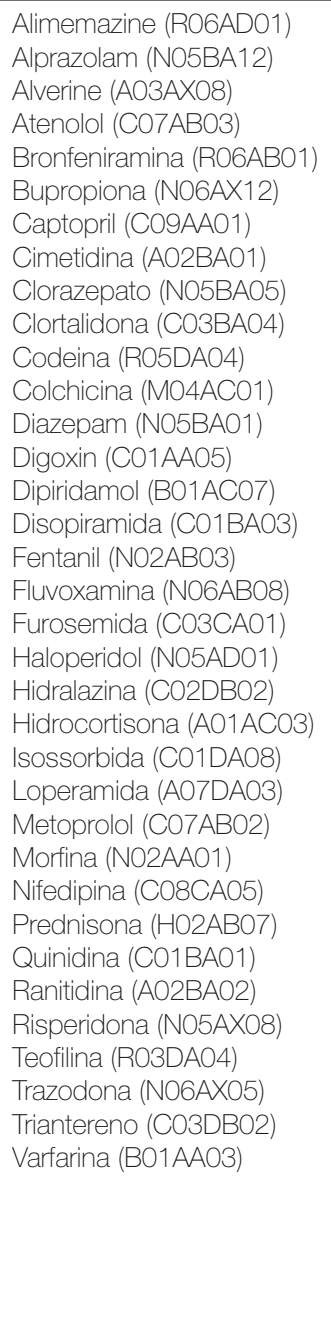 & $\begin{array}{l}\text { Alcalóides da beladona (A03BA04) } \\
\text { Amantadina (N04BB01) } \\
\text { Carbamazepina (N03AF01) } \\
\text { Ciclobenzaprina (M03BX08) } \\
\text { Ciproeptadina (R06AX02) } \\
\text { Levomepromazina (N05AA02) } \\
\text { Loxapina (N05AH01) } \\
\text { Molindona (N05AE02) } \\
\text { Oxcarbazepina (N03AF02) } \\
\text { Petidina (N02AB02) } \\
\text { Pimozida (N05AG02) }\end{array}$ & 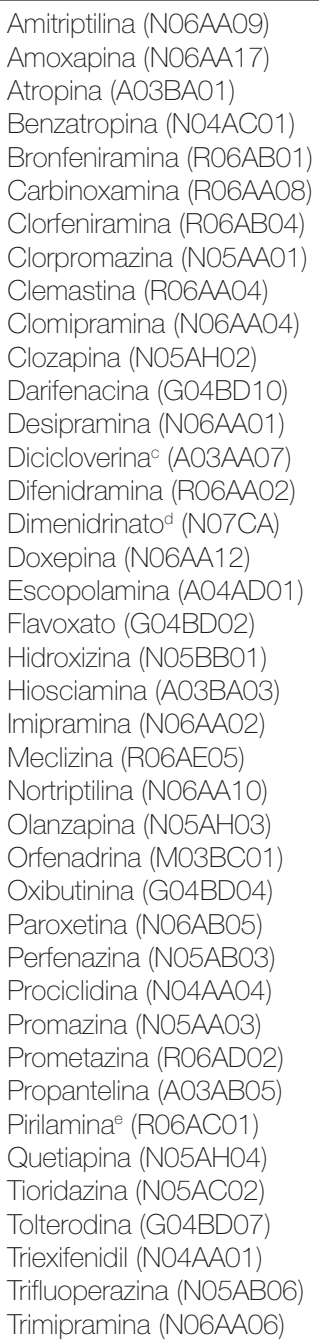 \\
\hline
\end{tabular}

Os fármacos são classificados conforme sua atividade anticolinérgica cognitiva (ACB): possível (escore 1), moderada (escore 2) e severa (escore 3). Os fármacos sem atividade relevante do ponto de vista clínico que, porém, apresentaram atividade anticolinérgica sérica ou afinidade in vitro por receptores muscarínicos são enquadrados na categoria "possível". Aqueles com evidências clínicas relevantes e estabelecidas quanto a efeitos adversos anticolinérgicos cognitivos são considerados dotados de atividade definida. Esta última constitui as categorias moderada e severa, 
conforme a habilidade de atravessar a barreira hematoencefálica e a associação com o desenvolvimento de delírio. Os fármacos não enquadrados em nenhuma categoria recebem escore zero. A pontuação resultante da soma dos escores dos fármacos utilizados por um mesmo indivíduo revela a ACB à qual o mesmo está sujeito. Escore total $\geq 3$ é considerado clinicamente relevante. Entre os medicamentos com escore 2, a Escala original inclui o Empracet (omitido neste Quadro); este é o nome comercial de um produto não comercializado no Brasil e que contém os princípios ativos paracetamol e codeína. ${ }^{a}$ Também conhecida como metotrimeprazina. ${ }^{b} \mathrm{Conhecida} \mathrm{como}$ meperidina. ${ }^{\circ}$ Conhecida como diciclomina. ${ }^{\circledR}$ Combinações de difenidramina teoclato (dimenidrinato) são classificados como NO7CA (preparações para vertigem). ${ }^{\circ} \mathrm{C}$ onhecida como mepiramina. ATC = Anatomical Therapeutic Chemical Classification System da Organização Mundial de Saúde (OMS). Fonte: Adaptado pela autora com base em Boustani et al. (2008). 

